\title{
COMPETENCIA Y PARTICIPACIÓN PRIVADA: LA EXPERIENCIA CHILENA EN EDUCACIÓN*
}

\author{
Osvaldo Larrañaga
}

El artículo revisa críticamente la literatura empírica respecto al impacto de la competencia y participación de privados en los resultados de la educación chilena. Se identifican períodos de acuerdo con la extensión de la competencia y participación privada y se discute la naturaleza de las pruebas de rendimientos que sirven de base a los estudios empíricos, así como la calidad metodológica de las investigaciones realizadas. De la evidencia empírica analizada se concluye que la competencia y la participación de privados han tenido un efecto positivo en los rendimientos escolares. Se advierte, no obstante, que estos resultados deben ser interpretados como logros relativos entre establecimientos, puesto que no se dispone de una medida absoluta de desempeño académico. Asimismo, se trataría de efectos de orden menor en relación a la brecha que presenta la educación en Chile respecto de países de similar grado de desarrollo económico y buenos logros educacionales.

Osvaldo Larrañaga. Ph. D. en Economía, Universidad de Pennsylvania. Ingeniero Comercial, Universidad de Chile. Profesor del Departamento de Economía de la Universidad de Chile.

* Este trabajo se originó como un artículo de referencia en el marco del proyecto Preal: "Políticas educacionales en Chile: Vouchers, Concentración, Incentivos y Rendimiento". Se agradecen los comentarios de Dante Contreras, Juan Pablo Valenzuela, Claudia Peirano y Joseph Ramos a versiones anteriores del trabajo, así como de un árbitro de Estudios Públicos. La responsabilidad definitiva del artículo y juicios emitidos sólo compete a su autor. 


\section{Introducción}

L

a experiencia chilena en educación ha tenido como característica distintiva la introducción de un esquema de competencia donde proveedores públicos y privados se financian con una subvención fiscal por alumno. Las características del sistema de educación chileno se aproximan a un esquema de vouchers, constituyendo un caso de estudio de interés internacional.

El análisis de la experiencia chilena es importante para el futuro diseño de la política educacional del propio país. Las pruebas internacionales de logros educacionales muestran que el país está muy por debajo de lograr estándares satisfactorios en su capital humano. La prueba TIMSS de 1999 situó al país en el lugar 35 de un total de 38 países participantes, por debajo de naciones con inferior ingreso per cápita (Mulis et al., 2000). La prueba SIALS de alfabetización de adultos mostró que más de la mitad de los chilenos entre 15 y 65 años son analfabetos funcionales, puesto que escasamente entienden lo que leen (Bravo, Contreras y Larrañaga, 2002).

Tal situación torna imperioso avanzar políticas públicas que induzcan cambios cualitativos en el nivel y la distribución de los resultados de la enseñanza en el país. La agenda de política debe estar basada en evidencia empírica sólida respecto de los determinantes efectivos de la calidad de la educación. El diseño de políticas sin base en el conocimiento sistematizado es análogo a buscar un objeto en una habitación oscura; sólo por casualidad y después de muchos esfuerzos fallidos podrá lograrse el objetivo deseado.

Este trabajo revisa críticamente las investigaciones empíricas que han evaluado el funcionamiento del sistema de competencia y participación privada de la educación en Chile. La revisión se centra en los estudios que utilizan los resultados de las pruebas estandarizadas de logros educacionales (SIMCE) como variable dependiente. Estas pruebas tienen un carácter prácticamente censal para los estudiantes de cuarto y octavo año de educación básica a partir de 1988. Otros trabajos relevantes han utilizado como variable de resultado a la prueba de selección a las universidades, cuyos contenidos y población objetivo poseen carácter más específicos.

El trabajo se organiza en cinco secciones principales. La primera sección caracteriza el grado de competencia y participación privada en el sistema educacional chileno. La segunda sección analiza el principal elemento de evaluación de la calidad de la educación en el país — las pruebas estandarizadas de rendimiento escolar SIMCE — presentando sus resultados principales y su comparación respecto de estándares internacionales. La 
tercera sección revisa la evidencia disponible respecto del efecto de la competencia entre establecimientos educacionales sobre los resultados del sector. La cuarta sección evalúa el rendimiento de los colegios particulares subvencionados en relación a los establecimientos municipales. Las conclusiones del trabajo se presentan en la última sección.

\section{Competencia y participación privada en el sistema chileno}

La evaluación de la experiencia chilena requiere caracterizar adecuadamente los elementos de competencia y participación privada allí presente. Sobre la materia hay discrepancias entre los expertos. Para algunos analistas el sistema educacional chileno ofrece una experiencia "de texto" de la aplicación de un esquema de vouchers (Hsieh y Urquiola, 2002). Para otros autores, el sistema contiene un conjunto de características que lo alejan de un modelo de vouchers (Aedo y Sapelli, 2001).

Resulta evidente que previo a derivar conclusiones respecto de la efectividad de la competencia y de la participación privada se precisa establecer cuándo y dónde se presentan estos factores en la experiencia chilena. En esta materia se distinguen tres períodos de interés: el impulso inicial (1980-1986); el período de baja intensidad de la competencia (1987-1994); y la reactivación de la competencia (1995 adelante).

\section{El impulso inicial: 1981-1986}

La experiencia chilena de competencia y participación privada se origina en los inicios de los 80. En esa época las antiguas escuelas públicas, dependientes del Ministerio de Educación, son transferidas a los gobiernos locales dando origen al sector municipalizado de educación. Al mismo tiempo, se autorizó a escuelas privadas a operar bajo financiamiento público con la condición que no cobraran pagos de matrícula a los estudiantes. Este sector de escuelas pasó a denominarse educación privada subvencionada.

Los administradores de las escuelas reciben de parte del Estado una subvención o pago por estudiante. La asignación de los recursos entre las escuelas sigue a las decisiones que las familias realizan respecto del lugar de estudios de sus hijos, en un contexto donde los oferentes educacionales compiten para atraer y retener a los estudiantes.

El sistema de la subvención se asemeja a un esquema de vouchers, aun cuando sea el Estado quien transfiere los recursos financieros a los 
establecimientos en función de la matrícula que asiste a clases. La subvención "sigue al alumno", como requiere el sistema de vouchers, pero los padres de familia pueden carecer del rol de clientes asertivos que imagina el esquema de subsidios a la demanda.

La reforma induce un fuerte cambio en el mercado de los profesores. Quienes trabajan en las escuelas municipales pierden su condición de empleados públicos, siendo transferidos a la normativa laboral del sector privado. Tal cambio significa que las condiciones de contratación y despido, así como la estructura de remuneraciones, pueden responder más cercanamente a los determinantes de oferta y demanda. Este es un requisito necesario para el funcionamiento del modelo de competencia en el sector de educación, considerando el peso de los salarios de los profesores en el costo de provisión del servicio.

La reforma tuvo un efecto dramático para la educación particular subvencionada. Alrededor de un $15 \%$ del total de los estudiantes del país emigran desde las escuelas públicas a las privadas subvencionadas en el período 1981-1986, causando un aumento de la participación en la matrícula del sector de $15,1 \%$ a $30,8 \%{ }^{1}$. El número de establecimientos del sector privado subvencionado crece en un $62 \%$ entre 1980 y 1985 . Por su parte, las escuelas públicas experimentan una caída en su participación de $78,0 \%$ a $63,1 \%$ en el período 1981-1986, aun cuando el número de sus establecimientos se mantiene relativamente constante (Cuadros A-1 a A-3 del Anexo).

En suma, este primer período se caracteriza por una fuerte competencia en el mercado educacional, originado en la introducción de la subvención como mecanismo de asignación de recursos, la flexibilización del régimen laboral de los profesores y la expansión de las escuelas privadas subvencionadas.

\section{El período de baja competencia: 1987-1994}

La etapa de rápido desarrollo inicial del modelo de competencia y participación privada fue de corta duración. Cambios en la situación económica y en la política educacional dan lugar a una segunda fase caracterizada por la detención del crecimiento del sector privado subvencionado y por la introducción de políticas que protegen a las escuelas municipales del rigor de la competencia. De esta manera, el período comprendido entre los

${ }^{1}$ El sector privado subvencionado existía en forma previa a la reforma de los 80 , tratándose de escuelas preferentemente religiosas que no estaban sujetas a un régimen competitivo con el sector público. 
años 1987 y 1994 se caracteriza por una reducción en la intensidad de la competencia.

El primer cambio es la fuerte reducción del gasto público en educación en el contexto de la crisis de la deuda externa de mediados de los 80 . Ello se materializa en la caída del gasto público en educación como porcentaje del PIB desde un 4,0\% en 1983 a una media de 2,8\% en los años 1987-1994.

La caída en el valor real de la subvención reduce el retorno a la inversión educacional y detiene la expansión del sector particular subvencionado. Quiroz y Chumacero (1997) realizan un estudio de costos y concluyen que los colegios privados subvencionados operaban prácticamente sin márgenes para la inversión, después de considerar el nivel de la subvención y los costos de la enseñanza.

La segunda modificación de importancia se origina en el cambio de la política educacional en 1990. En ese año se produce el retorno a la democracia y el nuevo gobierno da un giro a la política de educación de acuerdo con el diagnóstico de que los problemas de calidad y equidad no serían resueltos por los mecanismos de mercado (Cox y Lemaitre, 1999).

En este contexto se introduce un conjunto de políticas de carácter centralizado cuyo objetivo es incrementar los niveles de calidad y equidad de las escuelas, aun cuando la subvención sigue operando como principal instrumento de financiamiento en el sector.

En 1991 se dicta el Estatuto Docente, una normativa laboral que se aplica al sector de educación municipal y que tiende a generar inmovilidad en las plantas docentes de los establecimientos así como una estructura de remuneraciones alejada de las condiciones de mercado. La normativa es el resultado de la negociación política entre el nuevo gobierno democrático y el gremio de los profesores, el cual había experimentado un fuerte detrimento en sus condiciones económicas durante el anterior gobierno autoritario. En opinión de actores del proceso, el estatuto docente fue el precio que se pagó para no retrotraer el conjunto del sistema educacional a la situación pre-1980 (Cox y Lemaitre, 1999) ${ }^{2}$.

En el período 1987-1994 se detiene el crecimiento del sector privado subvencionado. El número de escuelas privadas subvencionadas se mantiene prácticamente constante (2.637 en 1994 versus 2.643 en 1985) tal como sucede con la participación del sector en la matrícula de estudiantes (32,9\% en 1994 versus 32,7\% en 1987).

${ }^{2}$ El gremio de profesores experimentó fuertes pérdidas en la condición laboral en el régimen autoritario, siendo además objeto de persecución política. 
La reactivación de la competencia: 1995-2002

Este último período se caracteriza por la reactivación de las condiciones de competencia, aun cuando las escuelas municipales siguen operando en un contexto de relativa protección. Un elemento clave es la introducción del financiamiento compartido, que consiste en un cobro que las escuelas privadas realizan a las familias en adición a la subvención que reciben del Estado. Ello genera las condiciones de rentabilidad que posibilita la reanudación de la inversión en este sector.

El esquema de financiamiento compartido se introduce en el año 1994 y se expande progresivamente durante los años siguientes. El mecanismo también está disponible para escuelas municipales de nivel medio, aun cuando el $89 \%$ de los colegios que cobraban financiamiento compartido el año 2000 eran particulares subvencionados. El cobro promedio ascendía en dicho año a $\$ 8.900$, cifra que representa un 53\% de la subvención estatal en el caso de enseñanza básica.

La introducción del esquema de financiamiento compartido explicaría el incremento en la oferta privada de colegios. Así, el número de establecimientos privados subvencionados aumenta en un $23 \%$ en el período 1995 a 2001, tras haber permanecido estancado durante el período previo. Correspondientemente, la matrícula del sector aumenta en este último período en un $29 \%$.

Estos datos sugieren que la dinámica del sector educacional habría estado fuertemente determinada por consideraciones de oferta vinculada al nivel de la subvención, un elemento que es generalmente obviado en los estudios que se centran en los factores de demanda.

Por otra parte, en el año 1995 se relajan algunas de las restricciones a la movilidad laboral, permitiéndose a los municipios modificar la dotación de profesores frente a cambios en la matrícula de estudiantes, sujeto a la condición que sean profesores que tengan contratos de carácter transitorio $^{3}$. Ese mismo año se introduce un esquema de incentivos (SNED) que favorece a los profesores de los colegios con mejores resultados medido a través de cuatro indicadores que combinan rendimiento, valor agregado e igualdad de oportunidades. Este esquema introduce una relación entre las remuneraciones de los profesores del sector municipal y sus logros, aun cuando se trata de un vínculo más bien débil ${ }^{4}$.

${ }^{3}$ Esto es, bajo el régimen de contratas. El esquema no puede aplicarse a quienes tienen contratos de planta.

${ }^{4}$ Cada dos años el $25 \%$ de las escuelas con mejor puntuación, al interior de grupos homogéneos, recibe un bono destinado a suplementar la renta de los profesores. En el período 2001-2002 el bono del SNED representó un 4,2\% del salario promedio de los profesores en el sector municipalizado (Mizala y Romaguera, 2001). 
También en 1995 se empiezan a publicar los resultados de las pruebas SIMCE a nivel de establecimiento. Ello representa un cambio estructural en la disponibilidad de información a disposición de los padres de familia, en el contexto de un sistema cuya eficacia requiere la toma de decisiones informadas por parte de sus actores.

\section{En síntesis}

El sector de educación en Chile combina elementos de mercado y de regulación estatal, siendo necesario distinguir entre períodos. Los mecanismos de competencia operan más libremente en la primera mitad durante los años 80. Posteriormente, la caída en el valor de la subvención y la introducción de políticas centralizadas restringe el ámbito de la competencia y de la participación privada. A partir de 1995 se produce una reactivación del funcionamiento de la competencia con la introducción del financiamiento compartido y la publicación de los resultados del SIMCE.

En la actualidad las escuelas privadas subvencionadas operan en un contexto competitivo, puesto que su financiamiento depende de las elecciones de las familias y porque poseen flexibilidad para ajustar la dotación y remuneraciones de los profesores. En cambio, el sistema municipal opera en un contexto más protegido, producto del estatuto docente y una restricción presupuestaria más "blanda". Ello, aun cuando está expuesto a la información pública de sus resultados y a la eventual competencia por parte de sostenedores privados.

\section{La calidad de la educación y los resultados de la prueba SIMCE}

La mayor parte de los estudios que analizan la experiencia chilena se basan en los resultados de la prueba SIMCE como medida de logros educativos. Corresponde por tanto conocer las características del instrumento de evaluación, previo al análisis de los estudios basados en el indicador.

a) Evaluando la medida de evaluación: ¿Qué mide la prueba SIMCE?5

El SIMCE es una prueba que mide logros en matemáticas y lenguaje para alumnos de cuarto y octavo grado en años alternativos. A

\footnotetext{
${ }^{5}$ Esta sección se basa en Eyzaguirre y Fontaine (1999).
} 
partir de 1998 se aplica también a los alumnos de segundo medio (décimo grado) ${ }^{6}$.

La característica más importante de la prueba SIMCE es su naturaleza relativa, cuyo objetivo es producir un orden (ranking) de los estudiantes antes que la evaluación de los logros educacionales según estándares educacionales establecidos (pruebas de criterio). Ello tiene implicancias importantes respecto del uso e interpretación que se otorga a los puntajes del SIMCE.

Así, los resultados del SIMCE no pueden interpretarse como una medida de logros educativos, puesto que la prueba no informa respecto del grado de aprendizaje de los contenidos pedagógicos de un determinado nivel de enseñanza. El puntaje promedio del SIMCE refleja el nivel de conocimientos del estudiante promedio, cualquiera que este sea. Se sigue que un "buen" puntaje SIMCE no es garantía de un "buen" nivel de calidad de la enseñanza.

Asimismo, no se puede otorgar interpretación cardinal a las diferencias de resultados en la prueba SIMCE. Tener el doble de puntaje de SIMCE no implica tener el doble de conocimientos, puesto que la variable tiene sólo sentido ordinal. Así también, que los resultados del SIMCE presenten una distribución normal no implica que los conocimientos estén igualmente distribuidos, puesto que la "función" que vincula a ambas variables no es lineal (metodología de construcción de las preguntas de la prueba). Ello también implica que una diferencia de diez puntos en la prueba puede tener distinta connotación en materia de conocimientos a lo largo de la distribución de puntajes SIMCE.

Una segunda característica importante es que las pruebas SIMCE del período 1988-1997 son no comparables en el tiempo. Ello responde a la metodología utilizada en su construcción. Ésta consiste en una selección de preguntas tal que se eliminan aquellas con respuestas correctas por debajo del $30 \%$ y arriba de $80 \%$, considerándose en definitiva un conjunto de preguntas tal que la media de respuestas correctas sea $65 \%$ en cuarto básico (60\% en octavo y segundo medio). Por tanto, el puntaje promedio debiera ser siempre $65 \%$ (60\%), si es que la muestra de alumnos que se utiliza para testear las preguntas fuese representativa de la población nacional ${ }^{7}$.

${ }^{6}$ En cuarto básico también se rinde una prueba de comprensión del medio, mientras que en octavo se rinden historia y geografía y ciencias naturales.

${ }^{7}$ El incremento de puntaje de las pruebas SIMCE en el período 1990-1996 se habría originado en la utilización de muestras de estudiantes con nivel de logros crecientemente inferior al promedio nacional. 
Por lo demás, las pruebas del período 1988-1997 se caracterizarían por problemas de calidad en su construcción. Eyzaguirre y Fontaine (1999) examinan las preguntas de la prueba de octavo básico de 1997, que por primera vez se hacían públicas, encontrando que las preguntas se caracterizaban por un bajo nivel de dificultad y mala calidad en la elaboración. De esta manera, el $78 \%$ de las preguntas de lenguaje de octavo básico correspondía a materias de tercero a sexto grado; la mayor parte de las preguntas eran simples e incluían "ayudas" para que el estudiante pudiera responder bien; existían ítems mal formulados cuyo poder de discriminación radicaba en la capacidad de entender la pregunta antes la evaluación de conocimientos.

A partir de 1997 se introduce la técnica de "equating", la cual permite la comparación de los resultados en el tiempo a través de la repetición de determinados ítems entre distintas pruebas, para luego correlacionar el puntaje entre estos ítems y el resto de las preguntas, y derivar así una medida del nivel de una prueba respecto de otra.

En el año 1998 la prueba SIMCE modifica sus contenidos, combinando materias de los currículos antiguo y reformado, e incorpora preguntas abiertas para medir destrezas y habilidades de tipo superior. Las preguntas son construidas por expertos y utilizan la teoría ítem respuesta, de modo que las preguntas más difíciles tienen mayor ponderación. De esta manera, se espera solucionar los problemas más evidentes de calidad de las preguntas, aun cuando la prueba sigue teniendo criterio ordinal.

\section{b) Los resultados de Chile en el contexto internacional}

Una adecuada caracterización de la calidad de la educación del país requiere la comparación de resultados con otros países. Más aún si las pruebas nacionales de logros son de naturaleza relativa, no informando del nivel efectivo para los resultados de la enseñanza.

A tal objetivo contribuye la participación de Chile en la prueba TIMSS de 1999 para alumnos de octavo grado. Ésta es una evaluación de logros educacionales en matemáticas y ciencias que cumple con los mejores estándares de calidad metodológica, permitiendo una comparación de los resultados entre los países participantes.

Los resultados revelan un problema de proporciones en la calidad de la enseñanza en el país. Así, Chile obtuvo el lugar 35 dentro de 38 países participantes en la prueba TIMSS de matemáticas. Los resultados muestran que sólo el $48 \%$ de alumnos de octavo básico en Chile alcanzan 
los estándares esperados para cuarto básico a nivel internacional en matemáticas (Mulis et al., 2000).

Un estudio semejante para niños de tercero y cuarto básico fue realizado para trece países de América Latina por la UNESCO. Los resultados muestran a Cuba muy distanciado del resto de los países de la región. En un segundo nivel compiten Chile, México, Brasil y Argentina. Sin embargo, estos países presentan un bajo valor de rendimiento: alrededor de un $40 \%$ de niños no entienden lo que leen, a la vez que el $85 \%$ no es capaz de resolver problemas de matemáticas del respectivo nivel de enseñanza (citado en Eyzaguirre y Fontaine, 2001).

Para efectos de referir los resultados del SIMCE a nivel internacional puede realizarse una homologación de los puntajes entre la prueba SIMCE de 1999 y la prueba TIMSS, para luego realizar una comparación con tres países participantes en esta última prueba y cuyo ingreso per cápita está inmediatamente por debajo de Chile: Malasia, Tailandia y Turquía ${ }^{8}$.

La homologación se facilita en la medida que las pruebas SIMCE y TIMSS ajustan sus puntajes a una función de distribución normal para cada uno de los países considerados. Ello posibilita comparar las respectivas distribuciones utilizando sólo la media y la desviación estándar.

Los resultados del ejercicio muestran que una brecha de 10 puntos en la prueba SIMCE (0,2 desviaciones estándares) corresponden a 12,3\% de la diferencia promedio del TIMSS entre Chile y Malasia. Las comparaciones con Tailandia y Turquía entregan diferencias de 20,9\% y 42,4\%, respectivamente (Cuadro A-7 del Anexo).

¿Qué factores pueden explicar el pobre logro educacional del país en relación a los estándares internacionales? Para responder este tipo de preguntas la base de datos del TIMSS incluye un gran número de variables que informan sobre el desarrollo económico y social de cada país, los recursos educacionales que cuentan los hogares y escuelas, el currículo de matemáticas, las actitudes y expectativas de los distintos agentes del sector, etc.

El Cuadro A-8 presenta el ranking que Chile ocupa en estos indicadores. Sorprendentemente, el país obtiene una mejor posición para cada uno de ellos, respecto de la obtenida en el resultado de la prueba. Puesto de otra forma, el país presenta peores resultados que los predichos por los determinantes observables de los logros educacionales 9 .

${ }^{8}$ No se consideró a los ex países socialistas por su particular relación entre capital humano e ingreso per cápita.

${ }^{9}$ Una conclusión similar se deriva del estudio de Beyer (2001), quien realiza un análisis econométrico de los resultados del TIMSS entre países para concluir que Chile está por debajo de los patrones esperados de logro, después de controlar por las variables socioeconómicas de relevancia. 
No son evidentes las razones que explican el bajo rendimiento de los estudiantes del país en el contexto internacional. Pero sí resulta claro que los factores de competencia y participación privada han mostrado baja efectividad para modificar la mala posición que presenta la educación chilena respecto de estándares internacional de calidad.

\section{Competencia y resultados de la educación: La evidencia empírica}

La introducción de un sistema de competencia en la educación (vouchers) tendría asociado efectos sobre la productividad de las escuelas y efectos de selección de los alumnos (Hoxby, 2002).

Los efectos sobre la productividad de los colegios se relacionan con los incentivos que genera la competencia sobre la oferta educativa. La competencia aumentará la productividad de las escuelas en la medida que se cumplan ciertas condiciones: que haya múltiples oferentes que compitan por las elecciones de las familias; que los presupuestos de los colegios dependan efectivamente de la elección de los padres; que los establecimientos educacionales puedan realizar una gestión autónoma; y que los padres de familia tomen decisiones racionales e informadas respecto de la educación de sus hijos.

Los efectos relacionados con la selección de los alumnos se originan en el hecho que el servicio de la educación depende de las características de sus usuarios. Así, los establecimientos educacionales pueden exhibir mejores resultados si consiguen mejores alumnos. A su vez, la elección de los colegios por parte de las familias tendrá en cuenta los atributos del establecimiento y las características de los estudiantes. Esta relación de elección bilateral origina agrupamientos de alumnos y colegios (sorting), con dos tipos de efectos sobre los resultados de la educación: (i) efectos relacionados con la composición de los estudiantes; (ii) externalidades asociadas a los efectos de los pares (peer effects).

\section{Consideraciones metodológicas}

La experiencia chilena ofrece un caso excepcional para evaluar los efectos de la competencia sobre los resultados educacionales. Sin embargo, no se dispone de información para realizar una evaluación antes/después de la introducción del sistema de competencia, puesto que las primeras prue- 
bas estandarizadas corresponden a un período posterior (pruebas PER de los años 1982 a 1984).

Los estudios que evalúan el efecto de la competencia han explotado las diferencias que exhibe la variable a nivel geográfico. Para tal efecto se utiliza la comuna, que es la unidad territorial administrada por los municipios. Chile tiene un total de 334 comunas, pero no todas ellas presentan un tamaño adecuado para que opere la competencia. Así por ejemplo, en 115 comunas hubo menos de 200 estudiantes rindiendo la prueba SIMCE de octavo básico el año $1999^{10}$. Por otra parte, en el caso de Santiago se precisa cuidar la asociación entre "comuna" y "mercado educacional", puesto que las 34 comunas que integran esta ciudad difícilmente constituyen mercados separados.

También es importante distinguir entre administradores de las escuelas y los establecimientos educacionales a su cargo, puesto que son los primeros quienes reciben los recursos de la subvención de acuerdo a la matrícula de los establecimientos que administran. La eventual disociación entre grado de competencia y número de escuelas puede ser particularmente relevante en el caso de las comunas grandes con baja participación de escuelas privadas, donde el municipio se asemeja a un monopolista del servicio educacional ${ }^{11}$.

La estimación econométrica del efecto de la competencia no requiere contar con un modelo estructural para los determinantes de los resultados educacionales. Basta con especificar las variables de control que son relevantes; esto es, aquellas que afectan los resultados educacionales y que varían conjuntamente con el grado de competencia ${ }^{12}$.

El problema más importante de la estimación se origina en la presencia de variables no observables que se correlacionan con la medida de competencia y con los resultados de la educación. En este caso el parámetro del efecto competencia será sesgado e inconsistente.

El tema es recurrente en los estudios de la materia. Típicamente, el grado de competencia se mide por la fracción de estudiantes que asiste a escuelas privadas en un mercado educacional. Ahora bien, la localización

${ }^{10}$ Estas comunas no sólo tienen espacio para unas pocas escuelas, sino que existe una baja probabilidad de entrada de escuelas privadas a comunas pequeñas (Auguste y Valenzuela, 2003).

${ }^{11}$ Los administradores de conglomerados de establecimientos pueden tener distintos esquemas de asignación interna de sus recursos. Podría argumentarse que, en último término, prevalecerá la lógica de la competencia, induciendo a los administradores a cerrar sus "sucursales" menos exitosas. Sin embargo, también puede existir una política de subsidios cruzados entre los establecimientos de una administración común. A la fecha no se dispone de estudios empíricos en la materia.

${ }^{12}$ Este es el denominado enfoque experimental. Véase Angrist y Krueger (1999). 
de las escuelas privadas en determinada área geográfica no es aleatoria, sino que responderá a las características que presenten las escuelas públicas y los estudiantes en dicha área. En la medida que se trate de características no observables - por tanto, no controlables-, se producirá un sesgo en el estimador del efecto de la competencia. Supóngase, a modo de ejemplo, que las escuelas privadas se localizan en áreas donde las escuelas públicas presentan mayores deficiencias en su (no observada) capacidad de gestión. Se sigue que existirá una correlación negativa entre competencia y resultados de las escuelas públicas, pero esta relación no corresponde a un efecto de la competencia.

Para tratar el problema descrito se pueden utilizar instrumentos; esto es, variables relacionadas con la competencia pero no así con los resultados de la educación. El instrumento provee variación exógena de la competencia, posibilitando la estimación consistente del parámetro de interés. Otra estrategia de estimación se basa en efectos fijos; la cual explota la variación temporal que presentan los resultados y las variables de interés. El supuesto metodológico es que los factores no observables relacionados con la competencia son fijos en el tiempo (por ejemplo, capacidad de gestión de las escuelas), de modo que se anulan en una ecuación de diferencias.

\section{Los estudios de competencia}

A la fecha se dispone de cuatro estudios que han evaluado el efecto de la competencia en los resultados de las pruebas SIMCE ${ }^{13}$.

El artículo de McEwan y Carnoy (1998) fue el primero que estudió el efecto de la competencia en el sistema educacional chileno, para lo cual utilizó los resultados de las pruebas SIMCE del período 1988 a 1996. La investigación se centró en estimar el impacto de la competencia en los logros de las escuelas públicas, que es uno de los efectos positivos esperados de un esquema de vouchers. La medida de la competencia fue el porcentaje de matrícula de colegios privados subvencionados en cada comuna.

${ }^{13}$ Contreras y Macías (2002) estudiaron los efectos de la competencia utilizando los resultados de la prueba de selección a las universidades (PAA). La PAA evaluaba habilidades verbales y matemáticas y era obligatoria para postular a la mayor parte de las universidades en Chile. A diferencia de la prueba SIMCE, la PAA era rendida por un subconjunto de los estudiantes: alrededor de dos tercios de la cohorte que egresaba de la educación secundaria (quienes a su vez representarían un $49 \%$ de la cohorte generacional). Las habilidades que medía la PAA dependían tanto de factores innatos como aquellos adquiridos en la familia y en la escuela.

Los resultados señalan que existiría una relación positiva entre la competencia a nivel comunal de los establecimientos y los resultados de la prueba de selección a las universidades. Los resultados generalizan a una variante donde el índice de concentración se desagrega entre colegios subvencionados y colegios privados pagados. 
Para tratar el problema de la endogeneidad de la medida de competencia los autores realizan una estimación con efectos fijos a nivel de establecimientos, suponiendo que los factores no observados se mantienen constantes en el tiempo. También utilizan constantes (efectos fijos) por año para tratar los problemas de comparición del SIMCE y los cambios en el nivel de la subvención ${ }^{14}$.

Los resultados del modelo con efectos fijos por colegio muestran un efecto negativo de la competencia: las comunas con mayor matrícula en colegios privados presentan colegios públicos con menor SIMCE (Cuadro $\mathrm{N}^{\circ}$ 1). Las estimaciones mínimo cuadrados presentan el resultado opuesto, que sería atribuible a un sesgo en los parámetros.

CUADRO N ${ }^{\circ}$ 1: $\quad$ EFECTO DE LA COMPETENCIA EN RESULTADOS SIMCE LENGUAJE, A NIVEL DE COLEGIOS (MCEWAN Y CARNOY)

Coeficientes estandarizados

OLS (mínimos cuadrados ordinarios)

OLS con controles comunales

Efectos aleatorios a nivel de escuela

Efectos fijos a nivel de escuela

Número de observaciones
$0,03 *$
$-0,04 *$
$-0,08 *$
$-0,16 *$

Alrededor de 15.000

Nota: Efectos de la competencia sobre rendimiento escuelas municipales, control por participación del colegio público en P-900, dummies por año.

Parámetros con asterisco son significativos a un 5\%.

Fuente: Basado en McEwan y Carnoy (1988).

Los autores argumentan que la relación negativa entre competencia y resultados de las escuelas públicas es causada por la emigración de los mejores estudiantes hacia las escuelas privadas. De esta manera, los colegios públicos localizados en comunas con mayor presencia de escuelas privadas obtienen puntajes más bajos. El resultado descrito podría reflejar tan sólo un efecto de composición, sin que necesariamente existan pérdidas netas a nivel de individuos. Tampoco excluye la posibilidad que la competencia en el sector de educación tenga algún efecto positivo sobre los

${ }^{14}$ La comparación a través de efectos fijos es válida a pesar que los resultados del SIMCE no sean comparables en el tiempo. Ello puesto que la metodología permite conocer si las escuelas bajo competencia ganan o pierden puntaje SIMCE respecto del resto de las escuelas. En cambio, no sabemos cómo evoluciona el rendimiento del conjunto de escuelas en el tiempo. Nótese también que la estandarización de los puntajes a través de una distribución normal facilita la comparación entre escuelas en distintos períodos de tiempo, puesto que se igualan las medias y varianzas de las respectivas distribuciones. 
colegios públicos, tan solo que cualquier impacto positivo sería dominado por el citado efecto de composición. Por otra parte, nótese que puede haber efectos de composición entre escuelas o entre comunas, a pesar que a nivel nacional la tasa de matrícula en las escuelas privadas subvencionadas se haya mantenido relativamente constante durante el período bajo análisis (Sección 1).

El segundo estudio corresponde a Hsieh y Urquiola (2002). El artículo se centra en el período 1982-1988, puesto que este presenta los mayores cambios de matrícula asociados a la introducción del sistema de competencia. Al igual que en el caso anterior, la medida de competencia utilizada en la participación de la matrícula del sector privado subvencionado y la metodología para tratar el problema de endogeneidad asociado es la estimación a través de efectos fijos.

Los resultados principales se presentan en dos niveles. Primero, se muestra que los resultados en las pruebas de matemáticas en los colegios públicos, en relación a los resultados de la respectiva comuna, disminuyen a medida que aumenta la presencia de escuelas privadas subvencionadas ${ }^{15}$. Los resultados obtenidos generalizan a otros dos indicadores de la educación - la tasa de repitencia y los años promedio de escolaridad de niños entre 10 y 15 años de edad—, así como a la composición socioeconómica de los alumnos (medida por la educación de los padres).

Estos resultados reflejarían el ya citado efecto de composición: el sistema de competencia induciría el traslado de niños con mejores características de aprendizaje a los colegios privados.

Un segundo nivel inquiere sobre la existencia de efectos a nivel agregado para cada comuna. La emigración hacia las escuelas privadas puede ser acompañada por externalidades positivas a nivel del sistema, como sería el caso del efecto dinámico de la competencia sobre las escuelas públicas. También pueden ocurrir externalidades de signo negativo, asociada al efecto de los pares (peer effect) si la salida de los mejores estudiantes afecta el resultado de quienes permanecen en las escuelas públicas $^{16}$.

Las estimaciones a nivel comunal no muestran efectos estadísticamente significativos en los coeficientes estimados (Cuadro $\mathrm{N}^{\circ} 2$ ). Se con-

\footnotetext{
${ }^{15}$ Los autores comparan los resultados de las pruebas PER y SIMCE, que se dispone en forma respectiva a inicios y fines del período analizado.

${ }^{16} \mathrm{El}$ efecto de los pares puede funcionar en distintas direcciones. Así, puede beneficiar a todos los estudiantes si los cursos más homogéneos son más efectivos para la enseñanza. También puede haber efectos distributivos, si un tipo de estudiante gana más que otros. La literatura no es definitiva respecto de estos efectos. Véase Hanushek et al. (2001); Hoxby (2000).
} 
CUADRO N ${ }^{\circ}$ 2: $\quad$ EFECTO DE LA COMPETENCIA EN PRUEBAS DE MATEMÁTICAS A NIVEL COMUNAL (HSIEH Y URQUIOLA)

Coeficientes estandarizados

\begin{tabular}{|c|c|c|}
\hline Controles & $\begin{array}{l}\text { Todas las comunas } \\
\text { con información }\end{array}$ & $\begin{array}{l}\text { Comunas urbanas, } \\
\text { excluye Santiago }\end{array}$ \\
\hline
\end{tabular}

$\begin{array}{lcr}\text { Ninguno } & 0,00 & 0,24 \\ \text { Socioeconómicos comuna } & -0,16^{*} & -0,08 \\ \text { Efectos fijos comunales } & -0,16 & 0,20 \\ & & \\ \text { Número observaciones } & 172 & 84\end{array}$

Nota: Panel de datos 1982-1988. Todas las regresiones contienen dummy para 1998. Parámetros con asterisco son significativos al 5\%.

Fuente: Hsieh y Urquiola (2002).

cluye que cualquier efecto neto de la competencia sobre la productividad de los colegios habría sido más que compensado por efectos de composición, incluyendo posibles externalidades negativas derivadas de la salida de los mejores estudiantes de los colegios públicos ${ }^{17}$.

En suma, los estudios descritos muestran la falta de efectos positivos de la introducción de la competencia sobre los resultados educacionales. El primero de estos estudios, McEwan y Carnoy (1998), así como otros trabajos de los mismos autores, habría entregado respaldo a la política pública que otorgó mayor peso a las intervenciones del Ministerio de Educación en la década de los 90.

El estudio de Gallegos (2002) tiene por objeto estimar los efectos de la competencia en el contexto de los incentivos que entrega el marco de política y la estructura del mercado. El trabajo está basado en los resultados del SIMCE del período 1994-1997. La estimación relaciona la variable competencia, medida como el porcentaje de matrícula privada a nivel de la comuna, con los resultados del SIMCE a nivel de establecimientos. Para tratar el problema de la competencia como variable endógena se utilizan como instrumentos la fracción de la población urbana y el número de estudiantes en la comuna. Estas variables se relacionan con la decisión de ofrecer educación en una comuna, pero no estarían correlacionados con los resultados de la educación.

${ }^{17}$ Los resultados del efecto de la competencia sobre las pruebas de rendimiento podrían estar sujetos a problemas de comparación, puesto que no todas las escuelas rindieron la prueba PER en 1982. Sin embargo, los resultados generalizan al caso de la tasa de repitencia y los años de escolaridad de la población entre 10 y 15 años, variables basadas en datos administrativos que no están sujetas a problemas de selección en la muestra. 
Considerando el conjunto de colegios subvencionados, los resultados muestran que la competencia contribuye a un mejor SIMCE del establecimiento promedio. El efecto de la competencia es mayor cuando sólo se utiliza una muestra de colegios privados subvencionados, sugiriendo que la estructura de incentivos importa puesto que los colegios privados están más expuestos a la competencia (Cuadro $\left.\mathrm{N}^{\circ} 3\right)$.

CUADRO N 3: $\quad$ EFECTOS DE LA COMPETENCIA EN SIMCE A NIVEL DE ESTABLECIMIENTOS (GALLEGOS)

Coeficientes estandarizados

\begin{tabular}{llllll}
\hline \multirow{2}{*}{ Colegios } & \multicolumn{2}{c}{ Cuarto básico } & & \multicolumn{2}{c}{ Octavo básico } \\
\cline { 6 - 7 } \cline { 5 - 6 } & 1994 & 1996 & & 1995 & 1997 \\
\hline Todos los subvencionados & $0,11^{*}$ & 0,03 & & $0,10^{*}$ & $0,07^{*}$ \\
Privados subvencionados & $0,17^{*}$ & $0,11^{*}$ & & $0,24^{*}$ & $0,20^{*}$ \\
Municipales & $0,09^{*}$ & 0,01 & & 0,06 & $0,04^{*}$ \\
\hline
\end{tabular}

Nota: Controles socioeconómicos. Asterisco indica parámetros significativos al 5\%.

Fuente: Basado en Gallegos (2002).

Finalmente, el trabajo de Auguste y Valenzuela (2003) analiza el efecto de la competencia sobre los resultados de la educación utilizando la base de datos de individuos de la prueba SIMCE del año 2000.

La estimación del modelo se basa en datos comunales ${ }^{18}$. Ello resuelve el problema de selección entre escuelas, suponiendo que las comunas representan mercados independientes. Al respecto, la ciudad de Santiago se trata como un solo mercado, pero los resultados son robustos a esta especificación. La variable competencia se mide por la participación del sector privado (pagado y subvencionado) en la comuna. Los autores realizan un cuidadoso tratamiento para la elección de instrumentos, mostrando que la población de la comuna y la distancia a la ciudad más cercana son instrumentos válidos ${ }^{19}$.

${ }^{18}$ Los autores construyen los promedios comunales a partir de datos individuales. Ello posibilita un mejor tratamiento estadístico de la materia, comparado con los demás estudios citados que se basan en datos a nivel de escuelas.

${ }^{19}$ En particular, se argumenta que el tamaño de la población es una medida del tamaño del mercado, que a su vez se relaciona positivamente con la presencia de escuelas privadas si se considera que la educación es una industria con producto diferenciado y sujeto a economías de escala. 
El Cuadro $\mathrm{N}^{\circ} 4$ presenta los principales resultados del estudio. Allí se muestra el cambio en la variable de resultado frente al cambio en la medida de competencia, ambos medidos en términos de desviaciones estándares. Los resultados se presentan para tres grupos de escuelas: todas, urbanas y urbanas subvencionadas por el Estado. Todas las regresiones utilizan como variables de control los promedios comunales del ingreso per cápita de las familias, la educación de las madres, el porcentaje de población indígena, así como un conjunto de variables mudas que controlan por el régimen de administración municipal y características geográficas.

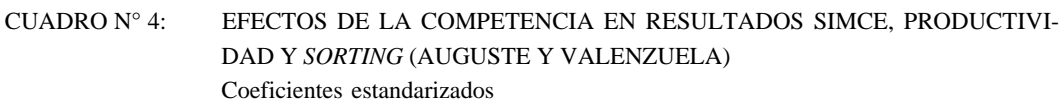

Número observaciones

254

221

219

Nota: Sorting se mide como la brecha comunal entre escuelas públicas y privadas, sea a nivel de resultados del SIMCE o de insumos (educación de la madre). Controles socioeconómicos y geográficos. Asterisco denota parámetros significativos a un $5 \%$.

Fuente: Basado en Auguste y Valenzuela (2003).

El primer conjunto de resultados concierne al efecto de la competencia sobre los puntajes de la prueba SIMCE, que ha sido la variable de interés de los estudios previos. Coincidiendo con Gallegos (2001), las estimaciones con variables instrumentales muestran que la competencia tiene un efecto positivo y significativo sobre los resultados del SIMCE. Para las escuelas urbanas financiadas por el Estado, un aumento de la medida de competencia de una desviación estándar causaría un cambio de 0.45 desviaciones estándares en el puntaje $\operatorname{SIMCE}^{20}$.

${ }^{20}$ Hay que tener presente que la desviación estándar del SIMCE a nivel comunal es cuatro veces menor respecto de la medición individual. 
Un segundo grupo de resultados considera como variable dependiente a la productividad de las escuelas, que se mide como el puntaje del SIMCE neto del efecto de las condicionantes socioeconómicas de los estudiantes $^{21}$. Los autores reportan el resultado para el conjunto de las escuelas urbanas, para las cuales un cambio de una desviación estándar en la competencia aumenta en 0.63 puntos la desviación estándar del SIMCE comunal.

El tercer grupo de resultados se refiere al efecto de la competencia sobre la distribución de estudiantes entre escuelas públicas y privadas, medida a través de resultados y de insumos. En ambos casos se considera la relación del promedio comunal de la variable entre las escuelas públicas y privadas. Los resultados muestran que la competencia aumenta la brecha entre las escuelas públicas y privadas, medidas a través de resultados o de insumos, validando la hipótesis que se produce una emigración de los mejores estudiantes hacia las escuelas privadas. Así por ejemplo, el aumento de una desviación estándar de la medida de competencia provocaría una caída de 0.78 puntos en el nivel socioeconómico de los alumnos de las escuelas públicas respecto de las privadas (medido por los años de educación de las madres, en desviaciones estándares). Este resultado es robusto a la metodología de regresión utilizada; mínimos cuadrados o variables instrumentales.

\section{¿Qué aprendemos de los estudios de competencia?}

La pregunta principal en la materia — si la competencia ha tenido un efecto positivo sobre la calidad de la educación - no puede tener una respuesta directa por falta de los datos requeridos a tal efecto. A la falta de información sobre resultados del proceso educacional en el período previo a la reforma, que podría haber posibilitado una evaluación "antes-después", se agrega la naturaleza ordinal de las pruebas de logros educativos de los años posteriores, que restringe la interpretación de los resultados obtenidos en los estudios que comparan los resultados entre escuelas o comunas sujetas a distinto grado de competencia.

De esta manera, los estudios existentes identifican efectos relativos de la competencia entre los establecimientos educacionales. Así por ejemplo, un mayor puntaje promedio del SIMCE en las comunas sujetas a mayor grado de competencia no demuestra necesariamente que la competencia

${ }^{21} \mathrm{La}$ variable dependiente son los coeficientes de variable dummies a nivel de cada comuna - y de la ciudad de Santiago- , estimados en una regresión de los datos individuales del puntaje SIMCE y que controla por las variables del hogar del estudiante. 
eleve el nivel de calidad de la enseñanza; simplemente plantea que esas comunas obtienen mejores logros en relación a otras comunas. En teoría, ambos tipos de comunas podrían estar en mejor (o peor) situación que en la situación sin competencia.

Los estudios establecen en forma sólida la existencia de un efecto composición, asociado a la migración de los mejores estudiantes desde los colegios públicos a los privados. Ello puede explicar una caída en el resultado SIMCE de los colegios municipales. Pero este es un efecto trivial y sin efecto sobre los resultados del sistema, a menos que existan externalidades negativas asociadas a la nueva composición de los pares en los colegios municipales. Esta es una pregunta sin respuesta aún definitiva.

A tal respecto, como lo señalan Aedo y Sapelli (2001) y Sapelli (2002), no se puede juzgar las bondades de un sistema de competencia si los "precios" que guían las decisiones de asignación de recursos son incorrectos. En particular, la selección es un resultado que se produce en la medida que la subvención no reconozca que es más caro educar a niños provenientes de hogares pobres o niños con problemas de aprendizaje. La solución radicaría en fijar precios correctos antes que eliminar el sistema de competencia ${ }^{22}$.

Los resultados de los estudios de brecha, que se reportan más adelante, muestran que los estudiantes que asisten a escuelas privadas subvencionadas obtienen mejores resultados en el SIMCE que sus contrapartes municipales, una vez que se controla por las variables socioeconómicas y por la elección del tipo de establecimiento educacional. No sabemos si ello es sólo causado por la mayor competencia a que está afecto el sector subvencionado - o si hay ventajas en la función de producción o si existe interacción entre competencia y tecnología- pero es a la postre un factor que relaciona competencia y logros educacionales. Esto es, si la competencia tiene asociada la expansión de las escuelas privadas, y si estas exhiben mejores resultados que las municipales, entonces existirá correspondencia entre competencia y logros educacionales.

El efecto neto de los distintos factores debiera ser observado en los resultados a nivel de comunas, recordando que se trata de resultados de carácter relativo. Así, la relación positiva entre competencia y logros educativos a nivel de promedios comunales, reportada en el estudio de Auguste y Valenzuela (2003), incorpora los diversos canales a través de los cuales actúa la competencia sobre los resultados educacionales. Hsieh y

${ }^{22}$ En el caso de un bien cuyo precio es fijado por debajo del equilibrio, tal situación sería análoga a suponer que la escasez resultante es producto de la competencia antes que causada por la política de precios. 
Urquiola (2002) mostraban el caso contrario utilizando datos comunales para el período 1982-1988, pero trabajan con datos de menor calidad y no estrictamente comparables. Las pruebas PER del período 1982-1984 cubren alrededor de la mitad de colegios que las pruebas SIMCE del año 1988.

El efecto de la competencia sobre la productividad debiera observarse en el sector privado subvencionado, que está más abierto a los incentivos de la elección de las familias y que tiene mayor espacio para modificar la tecnología de la enseñanza. A tal respecto, el estudio de Gallegos (2002) identifica que la competencia tiene efectivamente mayor impacto sobre el sector privado subvencionado. El parámetro del efecto de competencia es 3,5 veces más alto en el sector privado respecto del municipal. La magnitud del parámetro es significativa; por cada punto de desviación estándar que aumenta la participación privada, el resultado del SIMCE aumenta 0.244 unidades de desviación estándar en el caso de octavo básico (0.151 para cuarto básico).

En suma, la mejor evidencia sugiere que la competencia favorece los resultados educacionales. Sin embargo, se trata de un impacto relativamente menor, tal como lo señalan los autores de los respectivos estudios, y como lo confirma la comparación del país en el contexto internacional. Por otra parte, falta aún por conocer el funcionamiento de la competencia a nivel de sus diversos canales - efecto de composición y de selección, efecto de los pares, respuesta de los colegios públicos, etc.—, así como la distribución de sus resultados entre la población de estudiantes (versus sus efectos sobre los promedios).

\section{Educación pública versus privada}

La literatura nacional es relativamente prolífica en estudios que evalúan los resultados de las pruebas SIMCE entre escuelas municipales versus privadas subvencionadas. Si bien el propósito de estas investigaciones es realizar un estudio comparativo de la eficacia de las modalidades pública y privada de la oferta educativa, sus resultados también pueden ser interpretados como un test indirecto del sistema de vouchers, bajo la premisa que las escuelas privadas subvencionadas estarían más expuestas a los rigores de la competencia que sus contrapartes municipales.

Metodológicamente, los estudios de brecha entre escuelas se asimilan a la literatura del efecto tratamiento. En este caso, el tratamiento es asistir a un tipo de escuela (privada); mientras que la ausencia de tratamiento consiste en asistir al otro tipo de escuela (pública). La brecha entre 
tipos de escuela puede suponerse lineal y aditiva, resumiéndose en una variable muda, o puede considerarse un modelo que supone ecuaciones distintas según tipo de escuela, en cuyo caso la brecha puede ser evaluada en diferentes niveles de las variables de control.

Para evaluar los resultados de la educación privada versus pública hay que tener presente las potenciales fuentes de selección no aleatoria de estudiantes entre los establecimientos. Así, las familias más educadas y motivadas tenderán a elegir las mejores escuelas para sus hijos, a la vez que las escuelas tendrán incentivos a seleccionar a los mejores estudiantes a través de exámenes y procedimientos similares.

Bajo selección en variables observables, el efecto tratamiento puede ser calculado como la diferencia entre los resultados obtenidos en las pruebas SIMCE, condicional en las variables observables. Esto es, se compara el resultado educacional que consiguen estudiantes con características similares, salvo el tipo de escuela de asistencia.

El problema surge cuando la selección de las escuelas se realiza sobre la base de variables no observables, pero que se relacionan con los resultados educacionales (por ejemplo, motivación de los padres). Todos los estudios para Chile que han corregido por selección en las variables no observables han utilizado alguna variante del procedimiento de dos etapas de Heckman. En la primera etapa se estima una función de selección, que endogeniza la probabilidad de participar en el tratamiento. La segunda etapa estima la función de resultados, controlando por la probabilidad estimada de participar en el tratamiento. El supuesto clave de la metodología radica en asumir que los residuos de las distintas ecuaciones siguen una distribución normal conjunta ${ }^{23}$.

Los estudios realizados en el país se pueden separar en dos grupos de acuerdo a si utilizan información a nivel de establecimientos o de estudiantes. Tal característica está relacionada con la disponibilidad de la información. Los resultados del SIMCE estuvieron disponibles sólo a nivel de establecimientos en el período 1988-1997; mientras que a partir de 1998 se puede acceder a las bases de datos individuales.

\section{Resultados con datos a nivel de establecimientos}

En el primer grupo destaca por su carácter exhaustivo el estudio de Bravo, Contreras y Sanhueza (1999). Los autores utilizan todas las bases de datos de las pruebas SIMCE del período 1998-1997 para estimar la

${ }^{23}$ Véase Heckman (2001) para una revisión de las opciones metodológicas. 
brecha de rendimientos entre escuelas públicas y privadas subvencionadas, controlando por características socioeconómicas y geográficas. También se hace uso de los resultados de la prueba PER que fuera aplicada en los años 1982-1984 ${ }^{24}$. En cualquier caso no se controla por sesgo de selección, dado que se trabaja con resultados a nivel de establecimiento.

Los resultados principales se reportan en el Cuadro $\mathrm{N}^{\circ} 5$, diferenciándose según el tipo de controles utilizados: (i) ninguno; (ii) socioeconómicos, a través de variable categórica con cuatro niveles; (iii) geográficos, a partir de un índice construido sobre la base de tamaño de ciudad y dificultades de acceso.

CUADRO ${ }^{\circ}$ 5: $\quad$ BRECHA DE RESULTADOS ENTRE COLEGIOS PRIVADOS SUBVENCIONADOS Y MUNICIPALES. PRUEBA DE MATEMÁTICAS, CUARTO BÁSICO (BRAVO, CONTRERAS Y SANHUEZA) (Brecha por 1 sd puntaje SIMCE)

\begin{tabular}{|c|c|c|c|c|c|c|c|c|}
\hline Controles & 1982 & 1983 & 1984 & 1988 & 1990 & 1992 & 1994 & 1996 \\
\hline Ninguno & $0,22 *$ & $0,38^{*}$ & $0,41^{*}$ & $0,49^{*}$ & $0,50^{*}$ & $0,40 *$ & $0,43^{*}$ & $0,34 *$ \\
\hline Socioeconómico & $0,14^{*}$ & $0,27 *$ & $0,29 *$ & $0,20^{*}$ & $0,19 *$ & $0,18^{*}$ & $0,16^{*}$ & 0,14 \\
\hline $\begin{array}{l}\text { Socioeconómico, } \\
\text { geográficos }\end{array}$ & $0,11^{*}$ & $0,25^{*}$ & $0,29 *$ & $0,15^{*}$ & 0,03 & 0,06 & 0,12 & 0,04 \\
\hline Número escuelas & 2.815 & 3.017 & 3.291 & 5.513 & 4.421 & 4.162 & 4.522 & 5.110 \\
\hline
\end{tabular}

Nota: Todos los coeficientes con asterisco son significativos al $5 \%$. La media de las pruebas fluctúa en la cercanía de 50 y la desviación estándar en 10.

Fuente: Basado en Bravo, Contreras y Sanhueza (1999).

Los resultados se presentan en términos de tasas de la desviación estándar del SIMCE. Así, la brecha sin controles fluctúa entre 0.22 y 0.50 puntos de desviación estándar del SIMCE, a favor de los establecimientos privados subvencionados. Cuando se controla por factores socioeconómicos y geográficos, la brecha se reduce en alrededor de un tercio para las pruebas PER y a menos de la quinta parte en el caso de las pruebas SIMCE. De hecho, cuando se utilizan todos los controles la brecha desaparece, en sentido estadístico, en la mitad de las mediciones de la prueba SIMCE.

En el estudio de Mizala y Romaguera (1988) se estiman funciones de producción educacionales utilizando los datos de la prueba SIMCE del

${ }^{24}$ La prueba PER es un test estandarizado de logros en matemáticas y lenguaje que se introduce en 1982 a efectos de evaluar el sistema de vouchers. Sin embargo, la prueba se discontinuó en 1984 y sus resultados no fueron dados a conocer hasta 1995 por presiones internas en el Ministerio de Educación. 
año 1996 (cuarto básico) ${ }^{25}$. El resultado del SIMCE de cada establecimiento se regresiona en un vector de variables socioeconómicas (tramos de ingresos, índice vulnerabilidad), variables del establecimiento (experiencia profesores, razón profesor a alumno, número de colegios, área geográfica) y características de alumnos (asistencia a educación preescolar).

El resultado principal del artículo establece que no existen diferencias de puntaje SIMCE entre los colegios públicos y privados subvencionados, una vez que se controla por las variables descritas (Cuadro $\mathrm{N}^{\circ} 6$ ). También se reporta una apreciable disminución de la brecha de resultados entre establecimientos municipales y particular pagados.

CUADRO N ${ }^{\circ}$ 6: $\quad$ BRECHAS DE RESULTADOS ENTRE COLEGIOS MUNICIPALES Y PRIVADOS EN PRUEBA DE MATEMÁTICAS, CUARTO BÁSICO, 1996 (MIZALA Y ROMAGUERA) (Brecha por 1 sd puntaje SIMCE)

\begin{tabular}{lccccc}
\hline Controles & \multicolumn{2}{c}{$\begin{array}{c}\text { Brecha respecto de colegios } \\
\text { privados subvencionados }\end{array}$} & & \multicolumn{2}{c}{$\begin{array}{c}\text { Brecha respecto de colegios } \\
\text { privados pagados }\end{array}$} \\
\cline { 2 - 3 } \cline { 5 - 6 } & $\begin{array}{c}\text { Logros } \\
\text { SIMCE }\end{array}$ & $\begin{array}{c}\text { Valor } \\
\text { agregado }\end{array}$ & & $\begin{array}{c}\text { Logros } \\
\text { SIMCE }\end{array}$ & $\begin{array}{c}\text { Valor } \\
\text { agregado }\end{array}$ \\
\hline Ninguno & $0,41^{*}$ & $0,10^{*}$ & & $1,67^{*}$ & $0,44^{*}$ \\
Hogar & $0,06^{*}$ & 0,03 & & $0,65^{*}$ & $0,24^{*}$ \\
Hogar, geográficos & $-0,06^{*}$ & 0,02 & & $0,42^{*}$ & $0,17^{*}$ \\
Hogar, geográficos, insumos & 0,03 & 0,05 & & $0,45^{*}$ & $0,18^{*}$ \\
Número observaciones & 5.133 & 4.074 & & 5.133 & 4.074 \\
\hline
\end{tabular}

Nota: Parámetros con asterisco son significativos a un $5 \%$.

Fuente: Mizala y Romaguera (1998).

El estudio de Mizala y Romaguera (1988) representa el estado del arte del conocimiento de la materia hacia fines de los 90 . Sus conclusiones son citadas por personeros del Ministerio de Educación para mostrar que la modalidad de la escuela no hace una diferencia en el resultado educacional, una vez que se controlan las variables socioeconómicas, y que el mejoramiento de calidad de la educación debe ser objeto de intervenciones de política directa.

Tokman (2001) investiga la presencia de efectos diferenciales del tipo de escuela según características de los alumnos. Los datos utilizados son promedios por establecimientos correspondientes a la prueba SIMCE de 1996. Adicionalmente, se utiliza información socioeconómica proveniente

\footnotetext{
${ }^{25}$ Publicado posteriormente como Mizala y Romaguera (2000).
} 
de las encuestas CASEN de 1996 y 1998. El procedimiento es similar a Aedo y Larrañaga (1994), consistente en identificar en la encuesta de hogares estudiantes según escuelas, para luego asociar a cada establecimiento las características promedio del conjunto asociado de estudiantes ${ }^{26}$.

La estimación econométrica se realiza sobre la base de funciones distintas para escuelas públicas y privadas. Adicionalmente, se modela la elección de las escuelas a través de un modelo probit, en el contexto del método de dos etapas de Heckman.

Los resultados de la estimación con corrección por selección, bajo el supuesto de ventajas comparativas absolutas, muestra que la elección de las escuelas privadas se correlaciona negativamente con resultados del SIMCE (y la elección de escuelas públicas se relaciona positivamente). El resultado anterior se mantiene en el caso de las escuelas públicas cuando se admiten ventajas comparativas relativas, pero pierde significancia estadística para las escuelas privadas. Estos resultados son contrarios a los esperados y pueden estar influenciados por la selección de la muestra.

\section{Resultados con datos individuales}

Sapelli y Vial (2002) utilizan la base individual del SIMCE de 1998 para segundo medio y un modelo generalizado de elección de Roy ${ }^{27}$. Así, el estudio incorpora ecuaciones separadas del resultado del SIMCE para colegios públicos y privados, además de una ecuación de selección de colegios que depende de variables socioeconómicas y de variables que miden la disponibilidad de escuelas privadas a nivel de provincias (distrito electoral en el caso de Santiago). El supuesto aquí es que hay poca o nula movilidad entre estas áreas, de modo que se trata de variables que afectan la decisión de elección del colegio, no así los resultados de la prueba. El sesgo de selección se trabaja suponiendo que los errores de las tres ecuaciones siguen una distribución normal trivariada.

${ }^{26}$ El uso de la encuesta de hogares tiene asociado problemas. Primero, el tamaño de la "muestra" puede ser demasiado reducido para obtener buenos estimadores del factor socioeconómico de los establecimientos. Segundo, puede haber un sesgo de selección en la muestra de escuelas si se excluye a aquellas que no logran representación en la encuesta de hogares, lo cual depende del tamaño del establecimiento.

27 Este modelo hace explícita la elección que realizan las familias entre escuelas públicas o privadas, de acuerdo con los resultados esperados de la educación según características socioeconómicas e individuales de los alumnos. De este modo, las poblaciones que asisten a ambos tipos de escuelas diferirán según variables observables y no observables, cuyo adecuado control es necesario para inferir relaciones de causalidad entre el tipo de escuela y los resultados de la enseñanza. 
Controlando por sesgo de selección se obtiene un efecto de tamaño moderado a favor de la educación privada subvencionada, equivalente a un $15 \%$ de la desviación estándar (Cuadro $\mathrm{N}^{\circ} 7$ ). El resultado está referido al "tratamiento de los tratados"; esto es, quienes efectivamente asisten a escuelas privadas comparados con estudiantes de iguales características que asisten a escuelas públicas. De la ecuación de selección se desprende que habría ventajas absolutas antes que relativas (quienes van a colegios privados son "mejores" estudiantes que quienes asisten a establecimientos públi$\cos )$. Por otra parte, los resultados apuntan a una leve ventaja en favor de las escuelas públicas en el caso de individuos que se escogiesen en forma aleatoria en la población (efecto ATE).

CUADRO N ${ }^{\circ}$ 7: $\quad$ BRECHA COLEGIOS PRIVADOS SUBVENCIONADOS Y MUNICIPALES. DATOS INDIVIDUALES, SIMCE SEGUNDO MEDIO, 1998 (SAPELLI Y VIAL). (Efecto por 1 sd de puntaje SIMCE)

\begin{tabular}{lrrrrrr}
\hline & \multicolumn{6}{c}{ Tramos de ingreso familiar (miles de \$) } \\
\cline { 2 - 7 } Método & Todos & $<100$ & $100-200$ & $200-300$ & $300-600$ & $>600$ \\
\hline \multirow{2}{*}{ OLS, efecto ATE } & $0,20^{*}$ & $0,21^{*}$ & $0,21^{*}$ & $0,24^{*}$ & $0,18^{*}$ & $0,21^{*}$ \\
Heckman, efecto ATE & $-0,05^{*}$ & $-0,11^{*}$ & $-0,04^{*}$ & $0,01^{*}$ & $0,05^{*}$ & $0,01^{*}$ \\
Heckman, efecto TT & $0,15^{*}$ & $0,15^{*}$ & $0,16^{*}$ & $0,19^{*}$ & $0,12^{*}$ & $0,15^{*}$ \\
\hline
\end{tabular}

Nota: Todos los coeficientes con asterisco son significativos al 5\%. La media de las pruebas fluctúa en 250 y la desviación estándar 50.

Fuente: Basado en Sapelli y Vial (2002).

Mizala y Romaguera (2002) utilizan los datos individuales del SIMCE de 1998 (segundo medio) para estudiar la heterogeneidad de los resultados de las escuelas. Para tal efecto realizan estimaciones de las funciones de producción con regresiones de cuantiles. Un cuantil bajo (alto) representaría el caso de estudiantes con logros por debajo (arriba) del promedio, condicional en su situación socioeconómica y demás variables observables ${ }^{28}$.

Éste es uno de los pocos trabajos que analizan el efecto de los pares sobre el resultado educacional. La variable se define a nivel socioeconómico y del resultado del SIMCE, y se mide como el porcentaje de estudiantes

${ }^{28}$ La regresión de mínimos cuadrados tiene por objetivo ajustar una recta que minimice la distancia respecto del promedio de los datos observados. Las regresiones de cuantiles realizan igual procedimiento para determinados percentiles de los datos (por ejemplo, respecto de la mediana en el caso del percentil 50). 
por colegio que están en el rango de +/-0.5 desviaciones estándares alrededor de la media. Esta medida puede estar afecta a problemas, puesto que depende de la distribución de puntajes al interior del colegio. Así, es posible tener dos establecimientos con gran diferencia en la varianza de las variables, pero que entreguen igual valor para el efecto de los pares (tal como se mide en el artículo).

Los resultados revelan que el impacto del tipo de propiedad del establecimiento sobre el resultado educacional varía según los cuantiles de la distribución de no observables. Así, la brecha a favor de los colegios privados es mayor para los alumnos de cuantiles inferiores, disminuyendo para cuantiles superiores de la distribución de no observables (Cuadro $\mathrm{N}^{\circ} 8$ ). En todo caso, se constata una significativa brecha de puntajes a favor de los establecimientos privados subvencionados, después de controlar por los factores socioeconómico e insumos de la escuela.

CUADRO N ${ }^{\circ}$ 8: $\quad$ BRECHA COLEGIOS PRIVADOS SUBVENCIONADOS VS MUNICIPALES. REGRESIONES DE CUANTILES. SIMCE INDIVIDUAL SEGUNDO MEDIO 1998 (MIZALA Y ROMAGUERA) (Brecha por $1 \mathrm{sd}$ puntaje SIMCE)

\begin{tabular}{cccccc}
\hline OLS & Q 10 & Q 25 & Q 50 & Q 75 & Q 90 \\
$0,38^{*}$ & $0,48^{*}$ & $0,44 *$ & $0,38^{*}$ & $0,34^{*}$ & $0,30 *$ \\
\hline
\end{tabular}

Nota: Controla por factores socioeconómicos e insumos de colegios (función de producción). Todos los coeficientes con asterisco son significativos al 5\%. La media de las pruebas fluctúa en 250 y la desviación estándar 50.

Fuente: Mizala y Romaguera (2002).

Por su parte, el efecto de los pares socioeconómicos es más importante para los cuantiles bajos, sugiriendo que los estudiantes menos hábiles son quienes más ganan en los grupos homogéneos.

La interpretación de los autores alude a los residuos como una variable relacionada con las características del individuo (habilidades). Sin embargo, el residuo también puede responder a características de los establecimientos. Así por ejemplo, un colegio con mal liderazgo puede situar a gran parte de sus alumnos por debajo del resultado esperado del SIMCE (condicional en los observables). La distinción es importante a la hora de interpretar los resultados. La reducción de la brecha entre establecimientos públicos y privados para los cuantiles superiores puede tener distintas lecturas, según predominen factores individuales o de la escuela en los residuos. 


\section{¿Qué aprendemos de los estudios de brecha entre tipos de escuela?}

Los estudios de brecha ilustran la importancia que tienen los datos y las metodologías de estimación en las conclusiones de los trabajos. De esta manera, hacia fines de los 90 la conclusión dominante apuntaba a que la propiedad de los establecimientos no hacía diferencia en los resultados de las pruebas estandarizadas, una vez que se controlaba por los factores socioeconómicos de los estudiantes y por los insumos de los colegios.

Sin embargo, los estudios más recientes, basados en datos individuales, reportan resultados a favor de las escuelas privadas subvencionadas, después de controlar por las variables relevantes y por la decisión de selección de establecimientos. Así, Sapelli y Vial (2002) reportan una brecha de resultados a favor de las escuelas privadas subvencionadas equivalente a un $15 \%$ de la desviación estándar de la distribución de puntaje individual, un efecto significativo en el contexto nacional ${ }^{29}$.

Los resultados de estos estudios trascienden el ámbito de interés académico, constituyendo un factor de influencia en la política pública. La política pública de educación de los años 90, que privilegió las intervenciones centralizadas, encontró fundamentos en los trabajos que concluían que la competencia y la participación privada no tenían efecto sobre los resultados educacionales.

¿Qué explica los distintos resultados entre los estudios con datos de establecimientos versus aquellos basados en datos individuales?

Un primer elemento es la corrección del sesgo de selección que es posible realizar cuando se utilizan datos individuales. Como se plantea en Aedo y Sapelli (2001), la conclusión que la modalidad del establecimiento no importa después de controlar por el factor socioeconómico tendría validez si todo el efecto de esta variable estuviese asociado a una mejor capacidad de aprendizaje de los alumnos. La conclusión cambia si el factor so-

${ }^{29}$ Un resultado similar se encuentra en el estudio de Contreras (2002), basado en la prueba de selección de las universidades (PAA) del año 1998. La metodología de estimación tiene dos etapas. La primera etapa estima un modelo logit multinominal para la elección de escuelas privadas pagadas y privadas subvencionadas. En la segunda etapa se regresiona el logro en la PAA sobre edad, educación padres y la probabilidad estimada de asistir a la escuela respectiva. La brecha en la PAA de matemáticas de asistir a colegios subvencionados versus municipales es $0.11 \mathrm{sd}$ del puntaje de la prueba, de acuerdo a estimaciones OLS que controlan por la educación de los padres y la edad del alumno. El efecto sube a 0.37 puntos de desviación estándar en las regresiones en dos etapas que controlan por selección. La dirección del sesgo es contraria a la esperada si la selección de escuelas privadas se correlacionara positivamente con no observables que afectan los resultados de la PAA. La interpretación de los resultados se basa en el efecto marginal, esto es, en el efecto esperado para estudiantes en escuelas privadas pero que están restringidos por la disponibilidad de estas escuelas (estudiantes en comunas más pobres tienen más que ganar). 
cioeconómico está también asociado a una mejor elección del colegio, en cuyo caso la brecha de resultados responde a diferencias en la calidad de la educación que provee el establecimiento.

Una segunda materia a la hora de interpretar la brecha de resultados es el tratamiento de las variables de control. A tal respecto considérese que siempre existirá un número suficiente de controles que haga desaparecer la brecha entre los distintos tipos de establecimientos, puesto que se habrá dado cuenta de los factores que explican tal brecha. El punto relevante radica en la naturaleza endógena o exógena a los establecimientos educacionales de las variables que determinan los resultados de la enseñanza. Así por ejemplo, la diferencia a favor de los establecimientos privados que es explicada por la combinación de insumos educacionales responde a una decisión de política de los colegios, no correspondiendo controlar por tal variable cuando se evalúa la brecha de resultados entre establecimientos educacionales ${ }^{30}$.

Un tercer factor a considerar son los distintos resultados que se derivan de estudios basados en datos promedio por establecimiento versus datos individuales (Larrañaga, 2004). A tal efecto considérese el Cuadro $\mathrm{N}^{\circ}$ 9, el cual compara la brecha de resultados SIMCE entre establecimientos

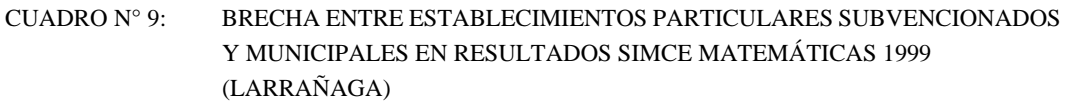

\begin{tabular}{lcc}
\hline Controles & Datos escuela & Datos individuales \\
\hline Ninguno & $17,98^{*}$ & $17,98^{*}$ \\
Hogar & 0,75 & $7,73^{*}$ \\
Hogar, escuela & 0,81 & $7,49 *$ \\
Hogar, escuela, región & $1,14^{*}$ & $8,40^{*}$ \\
No observaciones & & \\
Promedio SIMCE & 4.974 & 260.041 \\
Desviación estándar & 246,1 & 48,6 \\
\hline
\end{tabular}

Nota: Parámetros con asterisco son significativos a un 5\%; las estimaciones a nivel de establecimiento se ponderan por número de alumnos que rinden la prueba SIMCE; controles del hogar incluyen años de educación de padres y madres, ingreso y tamaño del hogar, los controles de la escuela son los recursos adicionales a la subvención vía financiamiento compartido o transferencia municipal.

Fuente: Larrañaga (2004).

${ }^{30}$ Este punto trasciende a si los datos son promedios por establecimiento o individuales. Sin embargo, es aplicable a algunos de los trabajos que utilizaron el primer tipo de información. 
públicos vs privados según se utilicen datos promedio por establecimiento o datos individuales. En ambos casos se trabaja con la misma fuente de datos primaria: los datos del SIMCE de matemáticas de 1999.

Los resultados difieren marcadamente entre los datos agregados y los datos individuales. Así, no hay brecha entre establecimientos cuando se controla por el factor socioeconómico y se utilizan datos agregados. En cambio, la brecha es significativa y a favor de las escuelas privadas subvencionadas cuando se utilizan datos individuales, más allá de los controles utilizados. Estos resultados replican aquellos reportados en los estudios de brecha que utilizaban uno u otro tipo de información.

Como se muestra en Larrañaga (2004), los parámetros provenientes de estimaciones basadas en datos individuales corresponden a un promedio ponderado de efectos intraescuelas y efectos interescuelas. Estos últimos corresponden a los parámetros reportados en los estudios basados en datos promedio por establecimiento. La divergencia de resultados según el tipo de datos utilizados refleja diferencias en el tratamiento de alumnos al interior de los establecimientos (efecto intraescuela) y en las prácticas de selección que ocurren entre escuelas (efectos interescuela). Así, existe menor diferencia de resultados del SIMCE entre alumnos de distinto nivel socioeconómico al interior de las escuelas privadas, a la vez que hay mayor diferencia de los resultados entre escuelas privadas con distinta población socioeconómica.

\section{Conclusiones}

La experiencia chilena de competencia y participación privada en educación debe ser apropiadamente contextualizada. La competencia opera en forma abierta en el sector de escuelas privadas subvencionadas, mientras que el sector municipal exhibe un conjunto de características que debilitan los mecanismos de la competencia, como es el caso de las restricciones presupuestarias blandas y la rigidez en la dotación y remuneración de sus profesores.

También es preciso distinguir entre períodos. Las restricciones a la competencia en el sector municipal son más importantes en la década de los 90. Desde mediados de los años 80 se produce una fuerte reducción del financiamiento público, que restringe la entrada de nuevos oferentes y la presión de la competencia. Esta situación se modifica a partir de 1995, cuando el financiamiento compartido posibilita la reanudación de la inversión educacional del sector privado subvencionado. 
La evaluación del efecto de la competencia en los resultados educacionales tiene como limitante la naturaleza relativa y no comparable en el tiempo de las pruebas SIMCE. En estricto rigor, no es posible conocer si la competencia ha elevado la productividad de las escuelas en Chile.

Las conclusiones de los estudios en la materia deben ser interpretadas como resultados relativos entre establecimientos. En este ámbito hay algunas constataciones principales.

Primero, durante la década de los 80 se produce una emigración de estudiantes con capacidad de aprendizaje superior al promedio desde las escuelas públicas a escuelas privadas. Ello origina un efecto de composición y reduce el logro relativo de las escuelas públicas; pero no está documentado si se producen efectos negativos netos por la acción de externalidades negativas relacionada con el efecto de los pares.

Segundo, los estudios que evalúan la brecha de resultados entre escuelas privadas subvencionadas y escuelas públicas favorecen a las primeras, después de controlar por las variables socioeconómicas y por la decisión de elección de establecimientos. La brecha a favor de las escuelas privadas es alrededor de un $15 \%$ de la desviación estándar de los puntajes de la prueba de rendimiento.

Tercero, la competencia afecta positivamente el rendimiento relativo de las escuelas privadas subvencionadas durante los años 90. Esto es, se verifica un efecto de la competencia cuando el contexto es el adecuado. El efecto es relevante: por cada punto que se eleva la competencia (medida en desviaciones estándares), los resultados del SIMCE crecen en 0,244 puntos de desviación estándar en octavo básico ( 0,151 en cuarto básico)

Cuarto, el efecto neto de los distintos factores se observa en los resultados a nivel de comunas. El estudio de Auguste y Valenzuela dispone de los mejores datos y reporta una relación positiva entre competencia y logros educativos a nivel de promedios comunales. En el caso de las escuelas urbanas, un aumento de la medida de competencia de una desviación estándar causaría un cambio de 0.45 desviaciones estándares en el puntaje SIMCE.

Los estudios de brecha ilustran la importancia de los datos para la derivación de conclusiones apropiadas. Así, cuando se disponía de datos a nivel de establecimientos (previo a 1998) los estudios de brecha tendían a concluir que no existían diferencias por tipo de establecimientos, después de controlar por la variable socioeconómica. De este modo, toda la diferencia de resultados entre establecimientos privados y públicos se explicaba por la distinta composición socioeconómica de los alumnos. 
La posterior disponibilidad de datos individuales permite controlar por la decisión de elección de establecimiento. En este caso se establece la existencia de la brecha a favor de los colegios privados.

Ello ocurre por dos tipos de razones. En primer lugar, los datos individuales permiten separar la influencia del factor socioeconómico en dos efectos - capacidad de aprendizaje de los estudiantes versus elección de los mejores colegios-, modificando el peso que se le atribuye a la variable para explicar las diferencias de resultados entre escuelas. En segundo término, las estimaciones basadas en datos agregados a nivel de establecimientos representan efectos interescuelas, mientras que aquellas basadas en datos individuales incluyen también efectos intraescuelas. La inclusión de estos últimos favorece a los establecimientos privados, puesto que presentan menor diferencia de resultados en su interior respecto de las escuelas públicas.

La pregunta más importante por responder a futuro son las razones de la gran brecha de resultados entre Chile y países de menor desarrollo económico, como es el caso de Malasia y Tailandia. La homologación de datos de las pruebas SIMCE y TIMSS permite concluir que la brecha de resultados entre las escuelas privadas y públicas nacionales representa menos de la décima parte de la brecha de resultados promedio entre Chile y Malasia. Resulta por tanto necesario identificar los factores que están detrás de esta segunda brecha.

$\mathrm{Al}$ respecto, no son evidentes las razones que explican el bajo rendimiento de los estudiantes del país en el contexto internacional. De hecho, el país muestra peores resultados que los predichos por los determinantes observables de los logros educacionales. En el supuesto de que el sistema educacional chileno presentase mayor grado de competencia y participación privada que la mayor parte de los demás países, se seguiría que este tipo de factores habría tenido baja efectividad para modificar la mala posición que presenta la educación chilena respecto de estándares internacionales de calidad. 


\begin{tabular}{|c|c|c|c|c|}
\hline \multirow{3}{*}{ CUADRO A-1: } & \multicolumn{3}{|c|}{ ANEXO } & \\
\hline & \multicolumn{4}{|c|}{ MATRÍCULA POR TIPO DE ESTABLECIMIENTO 1981-2001 (MILES) } \\
\hline & Total & Municipal & $\begin{array}{c}\text { Privado } \\
\text { subvencionado }\end{array}$ & Privado pagado \\
\hline 1981 & $2.841,7$ & $2.215,9$ & 430,2 & 195,5 \\
\hline 1983 & $2.869,4$ & $2.041,8$ & 643,8 & 183,8 \\
\hline 1985 & $2.963,4$ & $1.936,3$ & 832,4 & 194,7 \\
\hline 1987 & $2.962,7$ & $1.797,9$ & 968,6 & 196,2 \\
\hline 1989 & $2.976,0$ & $1.745,6$ & $1.012,7$ & 217,7 \\
\hline 1991 & $2.938,7$ & $1.698,8$ & $1.005,4$ & 234,4 \\
\hline 1993 & $3.020,1$ & $1.725,6$ & $1.025,3$ & 256,7 \\
\hline 1995 & $3.150,7$ & $1.777,7$ & $1.051,4$ & 282,7 \\
\hline 1997 & $3.306,6$ & $1.839,6$ & $1.155,5$ & 311,4 \\
\hline 1999 & $3.429,9$ & $1.866,9$ & $1.256,3$ & 306,5 \\
\hline 2001 & $3.559,0$ & $1.889,6$ & $1.356,4$ & 312,9 \\
\hline
\end{tabular}

Nota: Sector subvencionado incluye corporaciones educación técnico-profesional.

Fuente: Compendio Estadísticos MINEDUC, varios números.

CUADRO A-2: $\quad$ MATRÍCULA POR TIPO DE ESTABLECIMIENTO 1981-2001

(Composición porcentual)

\begin{tabular}{lcccc}
\hline & Total & \multicolumn{3}{c}{ Participación \% de la matrícula } \\
\cline { 3 - 5 } & & Municipal & Privado subvencionado & Privado pagado \\
\hline & & & & \\
1981 & $2.841,7$ & 78,0 & 15,1 & 6,9 \\
1983 & $2.869,4$ & 71,2 & 22,4 & 6,4 \\
1985 & $2.963,4$ & 65,3 & 28,1 & 6,6 \\
1987 & $2.962,7$ & 60,7 & 32,7 & 7,6 \\
1989 & $2.976,0$ & 58,7 & 34,0 & 8,0 \\
1991 & $2.938,7$ & 57,8 & 34,2 & 8,8 \\
1993 & $3.020,1$ & 57,4 & 34,1 & 9,2 \\
1995 & $3.150,7$ & 57,1 & 33,8 & 8,4 \\
1997 & $3.306,6$ & 55,6 & 34,9 & 8,8 \\
1999 & $3.429,9$ & 54,4 & 36,7 & \\
2001 & $3.559,0$ & 53,1 & 38,1 & \\
\hline
\end{tabular}

Nota: Sector subvencionado incluye corporaciones educación técnico profesional.

Fuente: Compendio Estadísticos MINEDUC, varios números. 
CUADRO A-3: $\quad$ NÚMERO DE ESTABLECIMIENTOS: 1980-2001

\begin{tabular}{rrrcr}
\hline & Total & Municipal & $\begin{array}{c}\text { Privado } \\
\text { subvencionado }\end{array}$ & Privado pagado \\
\hline 1980 & 8.799 & 6.370 & 1.627 & 802 \\
1985 & 9.787 & 6.476 & 2.643 & 668 \\
1990 & 9.741 & 6.288 & 2.694 & 759 \\
1995 & 10.302 & 6.422 & 2.822 & 1.058 \\
2001 & 10.589 & 6.242 & 3.460 & 1.031 \\
\hline
\end{tabular}

Nota: Excluye corporaciones técnico-profesionales.

Fuente: Compendio Estadísticos MINEDUC, varios números.

CUADRO A-4: $\quad$ GASTO PÚBLICO EN EDUCACIÓN 1981-2001

\begin{tabular}{lccc}
\hline & $\begin{array}{c}\text { Gasto por alumnos } \\
\text { en educación básica }\end{array}$ & $\begin{array}{c}\text { Gasto por alumnos } \\
\text { en educación media }\end{array}$ & $\begin{array}{c}\text { Gasto como \% } \\
\text { del PIB }\end{array}$ \\
\hline 1981 & 100,0 & 100,0 & \\
1983 & 91,0 & 89,7 & 4,3 \\
1985 & 89,7 & 90,3 & 4,0 \\
1987 & 83,9 & 70,9 & 3,0 \\
1989 & 85,9 & 72,7 & 2,6 \\
1991 & 91,7 & 72,1 & 2,6 \\
1993 & 114,1 & 100,0 & 2,8 \\
1995 & 142,9 & 129,7 & 2,9 \\
1997 & 150,0 & 180,6 & 3,4 \\
1999 & 174,8 & 194,2 & 4,0 \\
2001 & 196,6 & 226,0 & 4,4 \\
\hline
\end{tabular}

Fuente: Compendios de estadísticas del MINEDUC, varios años.

CUADRO A-5: MATRÍCULA SEGÚN TIPO DE ESTABLECIMIENTO E INGRESO FAMILIAR: COHORTE DE CUATRO BÁSICO EN 1999

\begin{tabular}{lrrrrr}
\hline Tipo de escuela & \multicolumn{5}{c}{ Nivel de ingreso familiar } \\
\cline { 2 - 5 } & 1 & 2 & 3 & 4 & Total \\
\hline Municipal & 50,3 & 32,5 & 13,2 & 3,0 & 100,0 \\
Privada subvencionada & 25,2 & 35,4 & 30,6 & 8,8 & 100,0 \\
Privada pagada & 0,6 & 1,9 & 15,6 & 82,0 & 100,0 \\
Total & 37,4 & 31,3 & 20,3 & 11,0 & 100,0
\end{tabular}

Fuente: Elaboración sobre base datos SIMCE 1999. 
CUADRO A-6: COBERTURA EDUCACIONAL POR QUINTILES DE INGRESO FAMILIAR (\% ASISTENCIA)

\begin{tabular}{|c|c|c|c|c|c|}
\hline \multirow[b]{2}{*}{ Edad } & \multicolumn{5}{|c|}{ Quintiles de ingreso del hogar (per cápita) } \\
\hline & 1 & 2 & 3 & 4 & 5 \\
\hline $4-5$ & 47,0 & 51,0 & 56,9 & 65,9 & 77,2 \\
\hline $6-13$ & 97,5 & 98,7 & 99,1 & 99,4 & 99,8 \\
\hline $14-17$ & 84,6 & 88,2 & 91,9 & 96,0 & 98,5 \\
\hline $20-24$ & 13,8 & 17,3 & 28,1 & 36,8 & 58,2 \\
\hline
\end{tabular}

Fuente: Cálculos sobre CASEN 2000.

CUADRO A-7: 10 PUNTOS EN LA PRUEBA SIMCE COMO \% DE BRECHA PROMEDIO EN PRUEBA TIMSS

\begin{tabular}{lcccc}
\hline & $\begin{array}{c}\text { TIMSS } \\
\text { promedio }\end{array}$ & $\begin{array}{c}\text { TIMSS desv } \\
\text { std }\end{array}$ & $\begin{array}{c}\text { Ingreso } \\
\text { p/c (ppp) }\end{array}$ & $\begin{array}{r}\text { 10 puntos SIMCE como \% } \\
\text { de brecha promedio TIMSS }\end{array}$ \\
\hline Chile & 392 & 85 & 12240 & 0 \\
Malasia & 519 & 81 & 7730 & 12,3 \\
Tailandia & 467 & 85 & 6490 & 20,9 \\
Turquía & 429 & 86 & 6470 & 42,4 \\
\hline
\end{tabular}

CUADRO A-8: RANKING DE CHILE EN TIMSS E INDICADORES ASOCIADOS

Puntaje prueba TIMSS

Pib per cápita (ppp, 1999)

Esperanza de vida al nacer

$13 / 36$

Tasa alfabetismo adulto

$24 / 38$

Gasto educación como \% pib

$27 / 37$

Recursos educacionales en casa \% alto

Expectativas de estudio \% estudiantes que esperan terminar universidad

$21 / 37$

Tiempo de estudio matemáticas fuera del colegio, promedio horas

$23 / 38$

(continúa) 
(continuación Cuadro A-8)

Índice autoconcepto de estudiantes en matemáticas (\% alto) 29/38

$\begin{array}{ll}\text { Índice actitud positivo hacia matemáticas (\% alto) } & 11 / 38\end{array}$

Métodos usados para apoyar implementación de currículo $\quad 37 / 37$

Tiempo de instrucción en matemáticas (\% del tiempo total) $\quad 7 / 32$

$\begin{array}{lr}\text { Acceso a internet en escuelas } & 18 / 36\end{array}$

Énfasis de profesores para tareas de matemáticas en casa (\% alto) 25/38

Disponibilidad recursos escuelas para enseñanza de matemáticas (\% alto) $\quad$ 16/38

$\begin{array}{ll}\text { Índice de buena asistencia a clases (\% alto) } & \text { 22/37 }\end{array}$

Interrupción de clases por indisciplina alumnos (\% no es serio problema) 25/36

$\begin{array}{ll}\text { Copia en clases ( } \% \text { donde no es serio problema) } & 10 / 37\end{array}$

Vandalismo (\% no es serio problema) $\quad 16 / 37$

Intimidación o abuso verbal a profesores (\% no es serio problema) 19/37

Dispone en casa: computador, escritorio, diccionario (\% los 3) 22/37

Número de libros en la casa (\% más de 10) 30/37

Padres con educación universitaria completa (\%) 23/36

Estudiantes que están de acuerdo con importancia de las matemáticas (\%) 1/38

$\begin{array}{ll}\text { Horas promedio de estudio } & \text { 24/37 }\end{array}$

$\begin{array}{lr}\text { Horas promedio de estudio en matemáticas } & 22 / 37\end{array}$

Índice "gusta" matemáticas $\quad$ 16/38

\% donde escuela ofrece "profundización" en matemáticas $32 / 38$

$\%$ donde escuela ofrece nivelación (remedial) en matemáticas $\quad$ 13/38

$\%$ con profesores cuya área principal de estudios es matemáticas $\quad$ 18/38

$\%$ con profesores que se sienten bien preparados en matemáticas $\quad 34 / 36$

\% escasez de profesores calificados en matemáticas (\% ninguna) 25/38

Número de días de enseñanza anual $\quad 18 / 37$

$\begin{array}{lr}\% \text { con test en clases (casi siempre) } & 2 / 38\end{array}$

$\begin{array}{lr}\text { Sin escasez de material de instrucción } & 9 / 38\end{array}$

Sin escasez de material de computación para enseñanza mat $\quad 10 / 38$

Fuente: Mulis et al. (2000). 


\section{REFERENCIAS BIBLIOGRÁFICAS}

Aedo, C. y O. Larrañaga: "Sistema de Entrega de los Servicios Sociales: La Experiencia Chilena". En C. Aedo y O. Larrañaga (eds.), Sistemas de Entrega de los Servicios Sociales: Una Agenda para la Reforma. Banco Interamericano de Desarrollo, 1994.

Aedo, C. y C. Sapelli: "El Sistema de Vouchers en Educación: Una Revisión de la Teoría y Evidencia Empírica para Chile". En Estudios Públicos 82, otoño 2001.

Angrist, J. y C. Krueger: "Empirical Strategies in Labor Economics". En O. Ashenfelter y D. Card (eds.): Handbook of Labor Economics, Vol. 3, Elsevier North Holland, 1999.

Auguste, Sebastian y Juan Pablo Valenzuela: "Do Students Benefit from School Competition? Evidence from Chile". Borrador, University of Michigan, 2003.

Beyer, H.: "Falencias Institucionales en Educación: Reflexiones a Propósito de los Resultados del Timss". En Estudios Públicos, 82, otoño 2001.

Bravo, D., D. Contreras y C. Sanhueza: "Rendimiento Escolar, Desigualdad y Brecha de Desempeño Privado/Público: Chile 1982-1997”. Documento de Trabajo, Departamento de Economía, Universidad de Chile, 1999.

Bravo, D., D. Contreras y O. Larrañaga: "Functional Literacy and Job Opportunities". Documento de Trabajo, Departamento de Economía, Universidad de Chile, 2002.

Brunner, J. J. y G. Elacqua: "Informe Capital Humano en Chile”. Universidad Adolfo Ibáñez, 18 de junio de 2003.

Contreras, D., L. Flores y F. Lobato: "Incentivos Monetarios a Docentes y Rendimiento Escolar. Evidencia para Chile”. Mimeo, Departamento de Economía, Universidad de Chile, 2002.

Contreras, D., y V. Macías: "Desigualdad Educacional en Chile: Geografía y Dependencia". En Cuadernos de Economía, Año 39, $\mathrm{N}^{\circ} 118$, diciembre de 2002.

Contreras, D., D. Bravo y C. Sanhueza: “PAA: ¿Una Prueba de Inteligencia?”. En Perspectivas, Vol. 4, $\mathrm{N}^{\circ} 2,2001$.

Contreras, D.: "Vouchers, School Choice and the Access to Higher Education". Economic Growth Center, Discusión Paper 845, Yale University, 2002.

Contreras, D., O. Larrañaga, L. Flores, F. Lobato y V. Macías: "Políticas Educacionales en Chile: Vouchers, Concentración, Incentivos y Rendimiento". Mimeo, Departamento de Economía, Universidad de Chile, 2003.

Cox, C., y M. J. Lemaitre: "Market and State Principles of Reforms in Chilean Education: Policies and Results". En G. Perry y D. Leipziger, Chile Recent Policy Lessons and Emerging Challenges. Washington D.C.: World Bank, 1999.

Epple, Dennis y Richard Romano: "Competition Between Private and Public Schools, Vouchers and Peer Group Effects". En AER 88 (1), 1998.

Eyzaguirre, B., y L. Fontaine: ¿Qué Mide Realmente el Simce?”. En Estudios Públicos, 75, invierno de 1999.

Eyzaguirre B., y L. Fontaine: "La Calidad de la Educación Chilena en Cifras". En Estudios Públicos, 84, primavera de 2001.

Fontaine, A.: "Equidad y Calidad en la Educación: Cinco Proposiciones Interrelacionadas". En Estudios Públicos, 87, invierno de 2002.

Fundación Nacional de Superación de la Pobreza: "Propuestas para la Futura Política Social". 1999.

Gallegos, F.: "Competencia y Resultados Educativos: Teoría y Evidencia para Chile”. En Cuadernos de Economía, Año 39, № 118, diciembre de 2002. 
Hanushek, E., y J. Luque: "Efficiency and Equity in Schools Around the World". Mimeo, 2001.

Hanushek, Eric, John Kain, Jacob Markman y Steven Rivkin: "Does Peer Ability Affect Student Achievement". NBER Working Paper 8502, October 2001.

Heckman, J.: "Micro Data, Heterogeneity and the Evaluation of Public Policy". En Journal of Political Economy, August 2001.

Hoxby, Caroline: "Peer Effects in the Classroom: Learning from Gender and Race Variation". NBER Working Paper 7887, August 2000.

Hoxby, Caroline: "Ideal Vouchers". Mimeo, Harvard University, 2002.

Hoxby, Caroline: "School Choice and School Competition: Evidence from the United States". Mimeo, Harvard University, 2003.

Hoxby, Caroline: "Introduction to The Economic Analysis of School Choice".

Hsieh, C., y M. Urquiola: "When Schools Compete, How do They Compete? An Assessment of Chile's Nationwide School Voucher Program". Mimeo, 2002.

Larrañaga, Osvaldo: "Socioeconomic Differences in the Achievement Gap between Public and Private Subsidized Education in Chile”. Documento de Trabajo $N^{\circ} 210$, Departamento de Economía, U. de Chile, noviembre de 2004.

McEwan, P. y M. Carnoy: "Competition and Sorting in Chile's Voucher System". Mimeo, Stanford University, 1988.

McEwan, P. y M. Carnoy: "The Effectiveness and Efficiency of Private Schools in Chile's Voucher System". Mimeo, 1999.

Ministerio de Educación: "Estadísticas de la Educación 2001". Santiago: Ministerio de Educación, 2002.

Mizala, A. y P. Romaguera: "Sistemas de Incentivos en Educación y la Experiencia del SNED en Chile". Documento de Trabajo No 82, CEA, Universidad de Chile, 2000.

Mizala, A. y P. Romaguera: "School Performance and Choice: The Chilean Experience". En Journal of Human Resources, Vol. XXXV, $\mathrm{N}^{\circ} 2$, Spring 2000.

Mizala, A. y P. Romaguera: "Regulación, Incentivos y Remuneraciones de los Profesores en Chile". Documento de Trabajo $\mathrm{N}^{\circ}$ 116, CEA, Universidad de Chile, 2001.

Mizala, A. y P. Romaguera: "Evaluación del Desempeño e Incentivos en la Educación Chilena". Cuadernos de Economía, Año 39, $\mathrm{N}^{\circ}$ 118, diciembre de 2002.

Mizala, A. y P. Romaguera: "Equity and Educational Performance". Documento de Trabajo $\mathrm{N}^{\circ}$ 136, CEA, Universidad de Chile, 2002.

Mullis, I. et al.: "Timss 1999. International Mathematics Report". ISC-IEA, 2000.

Núñez, J. e I. Millán: “¿Pueden Mejorar su PAA los Alumnos de Escasos Recursos? Evidencia Experimental”. Cuadernos de Economía, $\mathrm{N}^{\circ}$ 166, abril de 2002.

Quiroz, J. y R. Chumacero: "El Costo de la Educación Particular Subvencionada en Chile". Documento de Trabajo $\mathrm{N}^{\circ}$ 267, Centro de Estudios Públicos, 1997.

Sapelli, C.: "La Economía de la Educación y el Sistema Educativo Chileno. Introducción". En Cuadernos de Economía, Año 39, № 118, diciembre de 2002.

Sapelli, C. y B. Vial: "The Performance of Private and Public Schools in the Chilean Voucher System”. En Cuadernos de Economía, Año 39, № 118, diciembre de 2002.

Tokman, A.: "Is Private Education better?". En "Increasing Quality and Equity in Education: The Case of Chile". Tesis doctoral, Berkeley, 2001. 